Sādhanā Vol. 36, Part 6, December 2011, pp. 971-975. (C) Indian Academy of Sciences

\title{
An extension of Mangler transformation to a 3-D problem
}

\author{
$\mathrm{J} \mathrm{DEY}^{1, *}$ and A VASUDEVA MURTHY ${ }^{2}$ \\ ${ }^{1}$ Department of Aerospace Engineering, Indian Institute of Science, \\ Bangalore 560012, India \\ ${ }^{2}$ Tata Institute of Fundamental Research, Chikkabommasandra, GKVK Post, \\ Bangalore 560065, India \\ e-mail: jd@aero.iisc.ernet.in
}

MS received 29 October 2010; accepted 19 August 2011

\begin{abstract}
Considering the linearized boundary layer equations for threedimensional disturbances, a Mangler type transformation is used to reduce this case to an equivalent two-dimensional one.
\end{abstract}

Keywords. Boundary layer; three-dimensional; Mangler transformation.

\section{Introduction}

The Mangler transformation reduces an axisymmetric laminar boundary layer on a body of revolution to an equivalent planar boundary layer flow (Schlichting 1968). This transformation is also useful in turbulent boundary layer flow over a body of revolution (Cebeci \& Bradshaw 1968). Another application of this transformation is in the reduction of a laterally strained boundary layer to the Blasius flow (Ramesh et al 1997). In this case the span-wise velocity is zero along a streamline but its non-zero span-wise gradient appears as a source/sink term in the contunuity equation (Schlichting 1968). In this paper, we show that a Mangler type transformation can reduce a specific three-dimensional flow considered here to an equivalent two-dimensional case.

\section{Analysis}

Let $u^{*}, v^{*}$ and $w^{*}$ denote the non-dimensional velocity components in the non-dimensional $x, y$ and $z$ directions, respectively. $u_{0}$ and $v_{0}$ will denote the Blasius velocity components. The governing equations considered here are the linearized boundary layer equations for two- and three-diemnsional disturbances of Libby \& Fox (1964) and Luchini (1996). These authors perturbed the Blasius boundary layer as: $u^{*}=u_{0}(x, y)+u_{1}(x, y) \exp (i \alpha z), v^{*}=v_{0}(x, y)+$ $v_{1}(x, y) \exp (i \alpha z), w^{*}=w_{1}(x, y) \exp (i \alpha z)$; for 2-D flow $(z=0, w=0), u_{1}=u, v_{1}=v$. We first consider the two-dimensional case.

*For correspondence 


\subsection{2-D Case}

In this case, the governing boundary layer equations are (Libby \& Fox 1964),

$$
\begin{gathered}
\frac{\partial u}{\partial x}+\frac{\partial v}{\partial y}=0 \\
u_{o} \frac{\partial u}{\partial x}+v_{0} \frac{\partial u}{\partial y}+u \frac{\partial u_{o}}{\partial x}+v \frac{\partial u_{o}}{\partial y}=\frac{\partial^{2} u}{\partial y^{2}} .
\end{gathered}
$$

The boundary conditions are: $u(x, 0)=v(x, 0)=u(x, \infty)=(x, \infty)=0$. The Blasius boundary layer equations are,

$$
\begin{gathered}
\frac{\partial u_{o}}{\partial x}+\frac{\partial v_{o}}{\partial y}=0, \\
u_{o} \frac{\partial u_{o}}{\partial x}+v_{o} \frac{\partial u_{o}}{\partial y}=\frac{\partial^{2} u_{o}}{\partial y^{2}},
\end{gathered}
$$

along with the boundary conditions, $u_{0}(y=0)=v_{0}(y=0)=0, u_{0}(y \rightarrow \infty) \rightarrow 1$.

Adding and subtracting the quantity $\mathrm{u} / \mathrm{x}$ in the continuity eq. (1), we have

$$
\frac{\partial u}{\partial x}+\frac{\partial v}{\partial y}+\frac{u}{x}-\frac{u}{x}=0
$$

Consider the Mangler transformation,

$$
\begin{gathered}
X=\frac{x^{3}}{3}, Y=y x, u(x, y) \rightarrow U(X, Y) \\
V(X, Y)=\frac{1}{x}\left[\frac{y u}{x}+v\right], u_{o}(x, y) \rightarrow U_{o}(X, Y), V_{o}=\frac{1}{x}\left[\frac{y u_{o}}{x}+v_{o}\right] .
\end{gathered}
$$

The usual Mangler variables are $X, Y, U$ and $V$. The variables $U_{o}$ and $V_{o}$ are additional here. The boundary layer equations for an axi-symmetric body of radius $r$ differ from those for twodimensional flows by the term $(u / r)(d r / d x)$ in the continuity equation, $\frac{\partial(u r)}{\partial x}+\frac{\partial(v r)}{\partial y}=0$; for $r=x$, the term $(u / r)(d r / d x)$ becomes $u / x$, which acts as a source term in the continuity equation.

In terms of the variables in (6), the governing equations (1)-(4) become,

$$
\begin{gathered}
\frac{\partial U}{\partial X}+\frac{\partial V}{\partial Y}-\frac{U}{3 X}=0 \\
U_{o} \frac{\partial U}{\partial X}+V_{o} \frac{\partial U}{\partial Y}+U \frac{\partial U_{o}}{\partial X}+V \frac{\partial U_{o}}{\partial Y}=\frac{\partial^{2} U}{\partial Y^{2}}, \\
\frac{\partial U_{o}}{\partial X}+\frac{\partial V_{o}}{\partial Y}-\frac{U_{o}}{3 X}=0 \\
U_{o} \frac{\partial U_{o}}{\partial X}+V_{o} \frac{\partial U_{o}}{\partial Y}=\frac{\partial^{2} U_{o}}{\partial Y^{2}},
\end{gathered}
$$

respectively. 
The mean flow continuity eq. (9) now has an artificial sink term $U_{o} / 3 X$. However, the similarity variables $\eta=Y / \sqrt{3 X}, U_{o}=f^{\prime}(\eta)$ reduce the mean flow to the Blasius one; here, a prime denotes the derivative with respect to $\eta$. (This may be an interesting application of the Mangler transformation to the Blasius flow.)

In terms of the variables,

$$
U=3 X U_{1}(X, Y), V=3 X V_{1}(X, Y),
$$

equations (7) and (8) become

$$
\begin{gathered}
\frac{\partial U_{1}}{\partial X}+\frac{\partial V_{1}}{\partial Y}+\frac{2 U_{1}}{3 X}=0 \\
U_{o} \frac{\partial U_{1}}{\partial X}+V_{o} \frac{\partial U_{1}}{\partial Y}+\frac{U_{o} U_{1}}{X}+\left[U_{1} \frac{\partial U_{o}}{\partial X}+V_{1} \frac{\partial U_{o}}{\partial Y}\right]=\frac{\partial^{2} U_{1}}{\partial Y^{2}},
\end{gathered}
$$

respectively.

\subsection{3-D Case}

The governing boundary layer equations in this case are the linearized disturbance equations of Luchini (1996; his equations 7a-c),

$$
\begin{gathered}
\frac{\partial u}{\partial x}+\frac{\partial v}{\partial y}+w=0 \\
u_{o} \frac{\partial u}{\partial x}+v_{0} \frac{\partial u}{\partial y}+u \frac{\partial u_{o}}{\partial x}+v \frac{\partial u_{o}}{\partial y}=\frac{\partial^{2} u}{\partial y^{2}}, \\
u_{o} \frac{\partial w}{\partial x}+v_{o} \frac{\partial w}{\partial y}=\frac{\partial^{2} w}{\partial y^{2}} .
\end{gathered}
$$

( $i \alpha$ in Luchini's eq. (7a) is eliminated by taking $u_{1}=i \alpha u, v_{1}=i \alpha v, w=w_{1}$ ). The boudary conditions are: $u(x, 0)=v(x, 0)=w(x, 0)=u(x, \infty)=w(x, \infty)=0$. The third equation is the span-wise disturbance equation.

As in eq. (5), adding and subtracting the quantity $u / x$ in the continuity equation (14), we have

$$
\frac{\partial u}{\partial x}+\frac{\partial v}{\partial y}+\frac{u}{x}-\frac{u}{x}+w=0
$$

We consider the Mangler type transformation (6), along with an additional variable $W$, below

$$
\begin{gathered}
X=\frac{x^{3}}{3}, Y=y x, u(x, y) \rightarrow U(X, Y), V(X, Y)=\frac{1}{x}\left[\frac{y u}{x}+v\right] \\
W(X, Y)=\frac{1}{x^{2}}\left[w-\frac{u}{x}\right], u_{o}(x, y) \rightarrow U_{o}(X, Y), V_{o}=\frac{1}{x}\left[\frac{y u_{o}}{x}+v_{o}\right] .
\end{gathered}
$$

This additional variable $W$ is to relate the span-wise velocity component, $\mathrm{w}$, to the stream-wise velocity component, $\mathrm{u}$, as discussed below. In terms of these variables, the disturbance equations (14), (15) and (16) are,

$$
\frac{\partial U}{\partial X}+\frac{\partial V}{\partial Y}+W=0
$$




$$
\begin{gathered}
U_{o} \frac{\partial U}{\partial X}+V_{o} \frac{\partial U}{\partial Y}+U \frac{\partial U_{o}}{\partial X}+V \frac{\partial U_{o}}{\partial Y}=\frac{\partial^{2} U}{\partial Y^{2}} \\
2 U_{o} W+3 X\left[U_{o} \frac{\partial W}{\partial X}+V_{o} \frac{\partial W}{\partial Y}\right]+U_{o} \frac{\partial U}{\partial X}-\frac{U U_{o}}{3 X}+V_{o} \frac{\partial U}{\partial Y}=3 X \frac{\partial^{2} W}{\partial Y^{2}}+\frac{\partial^{2} U}{\partial Y^{2}},
\end{gathered}
$$

respectively.

Following Squire (1933), we add (20) and (21) to obtain

$$
\begin{gathered}
2 U_{o} W+3 X\left[U_{o} \frac{\partial W}{\partial X}+V_{o} \frac{\partial W}{\partial Y}\right]+2\left[U_{o} \frac{\partial U}{\partial X}+V_{o} \frac{\partial U}{\partial Y}\right] \\
-\frac{U U_{o}}{3 X}+U \frac{\partial U_{o}}{\partial X}+V \frac{\partial U_{o}}{\partial Y}=3 X \frac{\partial^{2} W}{\partial Y^{2}}+2 \frac{\partial^{2} U}{\partial Y^{2}} .
\end{gathered}
$$

In terms of the variables in (11), the continuity equation (19) and the momentum equation (22) become,

$$
\begin{gathered}
\frac{\partial U_{1}}{\partial X}+\frac{\partial V_{1}}{\partial Y}+\frac{U_{1}}{X}+\frac{W}{3 X}=0 \\
U_{o} \frac{\partial W}{\partial X}+V_{o} \frac{\partial W}{\partial Y}-\frac{U_{1} U_{o}}{3 X}+\frac{2 U_{o} W}{3 X}+2\left[U_{o} \frac{\partial U_{1}}{\partial X}+\frac{U_{o} U_{1}}{X}+V_{o} \frac{\partial U_{1}}{\partial Y}\right] \\
+U_{1} \frac{\partial U_{o}}{\partial X}+V_{1} \frac{\partial U_{o}}{\partial Y}=\frac{\partial^{2} W}{\partial Y^{2}}+2 \frac{\partial^{2} U_{1}}{\partial Y^{2}}
\end{gathered}
$$

respectively.

By letting $W=a U_{1}$ the disturbance equations (23) and (24) become,

$$
\begin{gathered}
\frac{\partial U_{1}}{\partial X}+\frac{\partial V_{1}}{\partial Y}+\frac{(3+a) U_{1}}{3 X}=0 \\
U_{o} \frac{\partial U_{1}}{\partial X}+V_{o} \frac{\partial U_{1}}{\partial Y}+\frac{(2 a+5)}{3(2+a)} \frac{U_{o} U_{1}}{X}+\frac{1}{(2+a)}\left[U_{1} \frac{\partial U_{o}}{\partial X}+V_{1} \frac{\partial U_{o}}{\partial Y}\right]=\frac{\partial^{2} U_{1}}{\partial Y^{2}},
\end{gathered}
$$

respectively. Comparing these with the two-dimensional equations (12) and (13), we find them similar. We may note that the span-wise velocity component, $w$, is $w=(1+a) u / x$. For $a=-1$, $\mathrm{w}=0$, equations (25) and (26) are exactly the same as (12) and (13), as it should be. Also, $\mathrm{u} / \mathrm{x}$ $(\sim w)$ acts as a source term in the continuity eq. (14). Thus enabling the use of the Mangler type transformation.

By letting $U_{1}=X^{N} g^{\prime}(\eta)$, and satisfying the continuity equation (25), the similarity form of (26) is readily obtained as,

$$
g^{\prime \prime \prime}+\frac{f g^{\prime \prime}}{2}+\frac{g f^{\prime \prime}}{2}+g f^{\prime \prime}\left[\frac{(7+a)}{2(2+a)}+\frac{3 N}{(2+a)}\right]-f^{\prime} g^{\prime}\left[3 N+\frac{(5+2 a)}{(2+a)}\right]=0 .
$$

In terms of the similarity variables, the perturbed velocity components are: $u=$ $3^{-N} x^{3(N+1)} g^{\prime}, w=(1+a) u / x$. 
For both (i) $a=-1, N=-4 / 3$ and (ii) $a=-8 / 3, N=-1 / 2$, eq. (27) reduces to the two-dimensional equation of Libby \& Fox (1964)

$$
g^{\prime \prime \prime}+\frac{f g^{\prime \prime}}{2}+\frac{g f^{\prime \prime}}{2}+f^{\prime} g^{\prime}-g f^{\prime \prime}=0
$$

The solution (Libby \& Fox 1964) of this equation is $g=f-\eta f^{\prime}$. The first case of $\mathrm{w}=0$ $(\mathrm{a}=-1)$ is obvious. In the second case, the perturbed velocities are: $u=3^{-1 / 2} x^{3 / 2} g^{\prime}$, and $w=-(5 / 3)(x / 3)^{1 / 2} g^{\prime}$. That is, the proposed Mangler type transformation (18) could reduce the three-dimensional problem considered here to a two-dimensional equivalent one.

\section{Conclusion}

A three-dimensional boundary layer flow is considered here. A Mangler type of transformation is proposed to reduce this flow to an equivalent two-dimensional one. This reduction has been possible by relating the span-wise velocity component to the stream-wise velocity component leading to an equivalent source term in the continuity equation.

\section{References}

Cebeci T, Bradshaw P 1968 Momentum transfer in boundary layers. Hemisphere, p 112

Libby P A, Fox H 1964 Some perturbation solutions in laminar boundary-layer theory, J. Fluid Mech. 17: 433

Luchini P 1996 Reynolds-number-independent instability of the boundary layer over a flat pate, J. Fluid Mech. 327: 101

Ramesh O N, Dey J, Prabhu A 1997 Transformation of a laterally diverging boundary layer flow to a two-dimensional boundary layer flow, Zeit. Angew. Math. Phys 48: 694

Schlichting H 1968 Boundary layer theory. McGraw Hill, p 605

Squire H B 1933 On the stability of three-dimensional disturbances of viscous fluid flow between parallel walls, Proc. Royal Soc. London A 142: 621-629 